\title{
Croissance industrielle et trame urbaine (Industrial growth and
}

\section{urban framework)}

Thérèse Saint-Julien

\section{Résumé}

Résumé. - A partir du cas particulier de la France de l'Ouest, l'auteur décrit les processus qui ont lié l'évolution de la distribution géographique des emplois industriels et celle de la configuration de la trame urbaine, au cours des 25 dernières années. La principale conclusion de cette communication est que le processus de croissance a avant tout favorisé la pérennité de la trame urbaine en place. Au contraire, la récession pourrait s'avérer beaucoup plus perturbatrice pour le système urbain.

\begin{abstract}
Abstract. - From the particular case of West France, the author describes the processe that have linked the evolution of the geographic distribution of industrial employments and the one of the shape of the urban framework over the past twenty five years. The main conclusion of this paper is that the process of growth has above all favoured the durability of the urban framework in existence. On the contrary, the recession might turn out to be much more disruptive for the urban system.
\end{abstract}

\section{Citer ce document / Cite this document :}

Saint-Julien Thérèse. Croissance industrielle et trame urbaine (Industrial growth and urban framework). In: Bulletin de l'Association de géographes français, $N^{\circ} 475-476$, 58e année, Janvier-février 1981. pp. 59-66;

doi : https://doi.org/10.3406/bagf.1981.5228

https://www.persee.fr/doc/bagf_0004-5322_1981_num_58_475_5228

Fichier pdf généré le 25/04/2018 


\title{
CROISSANCE INDUSTRIELLE ET TRAME URBAINE
}

INDUSTRIAL GROWTH AND URBAN FRAMEWORK

\begin{abstract}
RÉSUMÉ. - A partir du cas particulier de la France de l'Ouest, l'auteur décrit les processus qui ont lié l'évolution de la distribution géographique des emplois industriels et celle de la configuration de la trame urbaine, au cours des 25 dernières années. La principale conclusion de cette communication est que le processus de croissance a avant tout favorisé la pérennité de la trame urbaine en place. Au contraire, la récession pourrait s'avérer beaucoup plus perturbatrice pour le système urbain.

ABSTRACT. - From the particular case of West France, the author describes the processe that have linked the evolution of the geographic distribution of industrial employments and the one of the shape of the urban framework over the past twenty five years. The main conclusion of this paper is that the process of growth has above all favoured the durability of the urban framework in existence. On the contrary, the recession might turn out to be much more disruptive for the urban system.
\end{abstract}

Mots clés: France de l'Ouest, croissance industrielle, croissance urbaine.

Une importante question sous-jacente à la plupart des politiques d'aménagement du territoire qui ont été menées à l'échelle des espaces nationaux pendant la longue période de croissance que nous venons de connaître est restée assez mal résolue. Comment l'expansion industrielle s'est-elle répercutée sur la configuration spatiale du réseau urbain qui en est le support géographique essentiel ? La fin de la croissance, l'accentuation de la crise économique donnent à cette question une acuité plus grande encore. Comment se portent aujourd'hui sur la trame urbaine la stagnation voire la récession d'un certain nombre de branches industrielles? Selon que des reports s'effectuent de manière totalement aléatoire, ou qu'ils obéissent au contraire à certaines règles, leurs effets sur le réseau urbain sont différents et les moyens à mettre en œuvre pour les maîtriser le sont aussi.

Répondre complètement à cette vaste et importante question est une entreprise aujourd'hui un peu prématurée. Nous soumettons simplement à votre réflexion quelques éléments de réponse. Ils se fondent sur l'examen de l'évolution des grands établissements industriels des régions françaises de l'Ouest et du Centre-Ouest (Centre, Pays de la Loire, Bre-

- M.A. à l'Université de Paris I. 90, Boulevard du Maréchal-Joffre, 92340 BOURG LA REINE. 
tagne, Poitou-Charentes) entre 1961 et 1976 , en relation avec les transformations de la trame urbaine régionale. L'échantillon des grands établissements comprend toutes les unités qui ont atteint au moins une fois 500 salariés au cours de la période. En 1976, ces établissements concentrent 170000 salariés, soit $32 \%$ de l'effectif des salariés de l'industrie de ces régions. Ils y ont en 16 ans assuré plus de $11 \%$ de la croissance régionale de l'emploi non agricole, apparaissant ainsi comme un des fondements importants de leur assise industrielle (tableau 1). Notons par ailleurs que les régions ici regroupées se sont au cours de cette période, caractérisées par une croissance particulièrement rapide de l'emploi industriel (plus de 30 \% alors que la croissance nationale a été de $11 \%$ ) et aussi par un niveau de croissance urbaine qui les classe, de ce point de vue, dans le peloton des régions françaises les plus dynamiques.

Deux conclusions importantes nous semblent ici pouvoir être tirées. La première est que la tendance à la croissance favorise la conservation de la configuration géographique du réseau urbain, nous allons dire pourquoi. La seconde est que la récession semble globalement plus perturbatrice, ce dont nous allons nous expliquer dans un deuxième temps.

\section{COMMENT LA TENDANCE Ȧ LA CROISSANCE FAVORISE LA CONSERVATION DE LA CONFIGURATION GÉOGRAPHIQUE DU RÉSEAU URBAIN}

L'expérience montre que, toutes choses égales quant aux conditions technologiques, la croissance d'une activité peut correspondre à des expressions spatiales différentes suivant les choix qui sont faits en la matière. En effet, la croissance peut se traduire par un processus de concentration géographique, si l'on privilégie l'estimation des économies d'échelle et/ou d'agglomération. Elle peut au contraire, passer davantage par la démultiplication des points d'implantation. La trame s'enrichit alors de nouveaux noyaux. Pour un même nombre d'unités créées son maillage s'intensifie ou s'étend selon les distances établies entre les différents points. Cette alternative a, on le conçoit bien, des répercussions extrêmement différentes sur l'évolution de la trame urbaine en place. Dans le second cas, la croissance peut en outre avoir des effets extrêmement variés selon qu'elle se reporte de manière aléatoire et sans discrimination sur n'importe quel niveau de la hiérarchie urbaine, ou qu'elle se fixe au contraire de manière sélective sur certains types particuliers de villes. Par rapport à ces différents schémas, le cas de l'Ouest français peut être assez précisément situé et interprété.

Si l'on raisonne en termes de bilan, trois conclusions essentielles se dégagent. La première est que la proportion des actifs des différentes villes employés dans les grands établissements industriels a varié de manière assez similaire dans l'ensemble des agglomérations. La relation qui lie la taille des unités urbaines et l'importance de ces emplois industriels a conservé de 1961 à 1976 une intensité comparable. Cette permanence témoigne de l'assez grande ubiquité suivant laquelle la crois- 


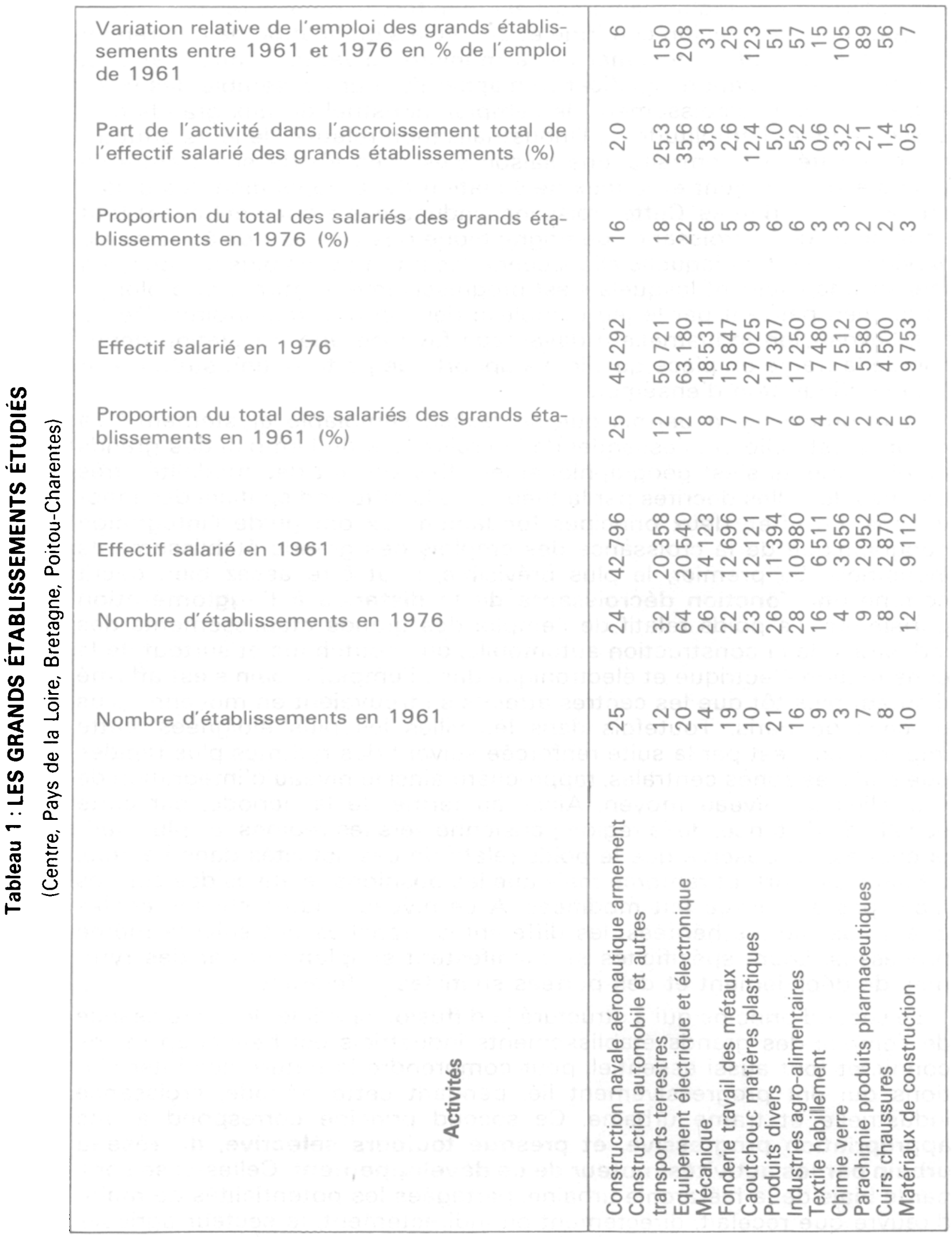


sance des grands établissements s'est manifestée dans le réseau urbain. La deuxième conclusion confirme et élargit la signification de ce premier constat. Elle peut se résumer de la manière suivante : entre 1961 et 1976, aucune relation significative n'apparaît pour l'ensemble des villes entre le taux d'accroissement de l'emploi industriel dû aux grands établissements et l'amplitude de l'industrialisation relative de leurs structures d'activité. Au contraire, une liaison s'est très tôt dessinée entre ce taux d'accroissement et le taux de variation de la population des unités urbaines considérées. Cette croissance industrielle a donc été avant tout un support de la croissance démographique des villes au-delà même de la période pendant laquelle elle a connu les rythmes les plus rapides; les mécanismes suivant lesquels s'est progressivement affirmé et prolongé ce soutien passant par le jeu complexe des apports migratoires. De ce fait, l'expansion industrielle a davantage favorisé une translation générale de la trame en place qu'elle n'a apporté de perturbation structurelle à sa configuration d'ensemble.

Quel processus a permis que se réalise semblable ajustement? La réponse est celle-ci : l'essentiel de la croissance de l'emploi des grands établissements s'est géographiquement fixé suivant des modalités très proches de celles décrites par la théorie de la diffusion spatiale des innovations. En effet, deux principes fondamentaux ont guidé l'intégration géographique de la croissance des emplois des grands établissements industriels. Le premier, le plus prévisible, peut être assez bien décrit comme une fonction décroissante de la distance à l'agglomération parisienne. Le poids relatif de l'emploi des grands établissements des industries de la construction automobile, du caoutchouc et surtout de la construction électrique et électronique dans l'emploi urbain s'est affirmé d'autant plus tôt que les centres atteints se trouvaient en moyenne plus proches de Paris. Toutefois dans les villes les plus éloignées, cette importance s'est par la suite renforcée suivant des rythmes plus rapides que dans les zones centrales, rapprochant ainsi le niveau d'intégration de ces villes du niveau moyen. Ainsi, au terme de la période, sur cette échelle de distance, de la région parisienne vers les régions les plus périphériques, on observe que le poids relatif de ces activités dans l'emploi urbain s'est partout renforcé mais que les positions relatives des centres n'ont pas été de ce fait modifiées. A ce niveau, toutes choses égales quant aux trames héritées, les différentes branches ont suivi le même processus. Leurs spécificités se manifestent simplement par des rythmes de déploiement et des portées spatiales différentes.

Le second principe qui a structuré la diffusion spatiale de la croissance de l'emploi des grands établissements industriels est beaucoup moins connu, et tout aussi essentiel, pour comprendre la nature des interrelations qui ont progressivement lié, pendant cette période, croissance industrielle et trame urbaine. Ce second principe correspond à une appropriation progressive, et presque toujours sélective, du réseau urbain par les activités moteur de ce développement. Celles-ci se sont, par le biais de la hiérarchie urbaine, partagées les potentialités de maind'œuvre que recelait, directement ou indirectement, le secteur agricole. 
Les modalités de ce partage sont intéressantes à examiner. Entre 1961 et 1976 , les grands établissements des industries du caoutchouc ont eu tendance a systématiquement accentuer leur emprise sur l'emploi des villes de taille moyenne. Pendant la même période on obsenve un relatif essaimage des établissements de l'industrie automobile vers ces mêmes villes moyennes. En réalité cet essaimage ne doit pas faire illusion. La construction automobile et les activités annexes renforcent leur influence sur les profils d'emploi des grandes villes entre 1961 et 1976 , reportant vers ce niveau de la hiérarchie urbaine une part extrêmement importante de la croissance de leurs effectifs salariés. Ces activités contribuent plutôt, au début comme à la fin de la période à fonder la spécificité des profils d'activité des grandes villes, en dépit de leur relative diffusion dans les centres de moindre importance. Enfin en 1961, on peut aussi considérer les industries d'équipement électrique et électronique comme des industries plutôt représentatives des profils d'emploi des unités urbaines d'assez grande taille. Les unités de production de cette activité y représentent alors une proportion beaucoup plus importante qu'ailleurs de l'ensemble des grands établissements industriels, et son taux de pénétration dans l'emploi urbain y est aussi plus développé. Progressivement, la force de cette emprise relative s'est déplacée des grandes villes vers les centres de moindre importance. La diffusion des salariés de cette branche dans les villes s'est donc pour une large part effectuée en suivant les canaux de la hiérarchie urbaine. Pour aucune de ces activités, la diffusion de la croissance ne s'est donc reportée de manière aléatoire sur le réseau urbain.

Et si l'on considère non plus les spécificités de ces processus de report mais leur combinaison, on constate que tout s'est passé comme si, au cours de la période, un certain partage du système urbain s'était progressivement réalisé entre ces activités de main-d'œuvre, les différents niveaux de la hiérarchie urbaine ayant été sélectivement ou successivement investis par chacune d'elles. Sans doute les trames initiales des trois branches se sont-elles profondément transformées mais les processus de diffusion se sont développés de telle manière que leur combinaison favorise d'abord la pérennisation de la forme générale de la trame urbaine régionale et n'entraîne que secondairement un rapprochement de l'ensemble des structures fonctionnelles.

On ne peut cependant réduire la croissance des grands établissements industriels et donc leur impact sur la restructuration de l'espace interurbain a l'évolution de ces activités motrices et l'on ne saurait passer sous silence le rôle de branches dont l'expansion a été moins spectaculaire, mais qui ont cependant assuré près du quart de la croissance d'ensemble (exemple : industries mécaniques, industries agro-alimentaires etc.). Les modalités suivant lesquelles ces branches renforcent dans les villes leurs positions relatives témoignent d'une moindre maîtrise de l'espace et d'une certaine dépendance à l'égard des stratégies géographiques des activités plus dominantes en la matière. Nous avons ainsi interprété la relative convergence de l'essentiel de la croissance de ces branches vers des villes qui sont en général de petite taille. Cette convergence vers les petites agglomérations ne fait que contribuer à la régularité de l'innervation du réseau urbain par la croissance industrielle récente.

En conclusion, on ne peut comprendre que la configuration de cette trame urbaine régionale ait subi si peu de bouleversements, en dépit 
d'une expansion industrielle sans précédent depuis le début du siècle, que si l'on tient compte de toute la complexité qu'a revêtu au cours de ces deux décennies le processus de diffusion spatiale de la croissance industrielle. Il faut à cet égard donner sa juste place à la complémentarité des pratiques spatiales qui s'est tacitement et progressivement instaurée entre les différentes branches à l'action au cours de cette phase peut être un peu particulière de l'histoire urbaine.

\section{LA RÉCESSION SERAIT-ELLE GLOBALEMENT PLUS PERTURBATRICE?}

Nous livrons ici, plus une question qu'une affirmation, des indices d'un processus plus que les composantes du processus lui-même. En effet, si la conjoncture de croissance s'est maintenue sans grands accrocs pendant assez longtemps, la situation de crise ne s'est dessinée que depuis un nombre restreint d'années. Notre attention a cependant été retenue par le fait que certaines branches se sont beaucoup plus tôt que d'autres installées dans des conjonctures de récession. L'observation de leur comportement spatial dans cette récession nous a paru de nature à éclairer certaines évolutions présentes.

Considérons d'abord l'agrégat qui regroupe les industries de la construction navale, de l'aéronautique et de l'armement. Le taux de croissance de l'emploi régional de ces grands établissements n'a été pendant ces 16 années que très légèrement positif $(+6 \%)$ et le solde du nombre de ces établissements très légèrement négatif alors que pendant le même temps les industries de l'aéronautique et de l'armement en particulier ont connu en moyenne en France une croissance beaucoup plus importante. Nous considérons donc que cet ensemble régional est plus significatif de la stagnation que de l'expansion. Cette évolution se matérialise par une rétraction spatiale qui se réalise, pour au moins deux raisons au bénéfice des points traditionnellement les plus forts. La croissance de la période s'est presque intégralement reportée sur les points autour desquels s'étaient déjà antérieurement constituées les plus grandes concentrations géographiques. Ce sont par ailleurs les établissements industriels géographiquement les plus isolés, les plus périphériques, qui ont le plus mal résisté et montré les signes les plus évidents de ralentissement, voire de déclin. On conçoit bien que les modalités de ce processus de rétraction soient indissociables des importantes immobilisations que nécessitent, dans l'ensemble, ces activités et de l'appartenance de chacune d'elle à des secteurs économiquement très concentrée. Le processus de rétraction impose donc bien à la trame urbaine une logique qui est propre à la spécificité des trames industrielles en place et qui contribue à l'accentuation des disparités interurbaines existantes.

Tout aussi perturbateur dans son principe pour la trame urbaine, le reflux des industries du textile et de l'habillement reflète l'existence d'interdépendances d'un tout autre ordre entre les deux systèmes. Entre le début des années 60 et 1976 ces activités perdent dans l'ensemble 
de la France $22 \%$ de leurs salariés; dans le même temps, le nombre de leurs grands établissements est dans l'Ouest, multiplié par 2 et l'effectif employé augmenté de $15 \%$. Ce bilan vérifie s'il en était besoin que ces régions ont été pour ces activités en crise un espace de repli, mais très provisoirement. Il cache en effet une évolution plus complexe qui combine une croissance très rapide au cours d'une phase assez courte (1965-1969), suivie d'un déclin lent et continu qui ramène en 1976 le volume total des emplois à ce qu'il était en 1965. Or on observe que le déclin de ces activités ne se manifeste pas de manière aléatoire dans la trame urbaine régionale. L'amplitude du déclin est en moyenne inversement proportionnelle à la distance à l'agglomération parisienne. Au-delà de 400 kilomètres, il n'y a plus déclin mais plutôt légère expansion des unités en place jusqu'en 1976. Nous avons par ailleurs mis en évidence que ce processus de déclin est apparu d'abord dans les plus grandes villes et n'a atteint que plus tardivement les unités urbaines de moindre taille, tout se passant comme si la régression s'était propagée du sommet vers la base de la hiérarchie urbaine. Que les établissements qui ont le plus tôt amorcé ce mouvement de reflux soient aussi ceux dont le taux de féminisation de la main-d'œuvre était le plus faible et qui donc se devaient de maintenir en moyenne un niveau des salaires plus élevé est alors bien dans la logique des choses. Ce processus de rétraction montre l'extrême fragilité d'une telle intégration régionale et surtout l'extrême sensibilité de ces activités aux capacités différentielles qu'offrent en matière de main-d'œuvre les différents niveaux de la hiérarchie urbaine. Tendant à renforcer l'emprise relative de ces activités en déclin sur les villes périphériques, il concourt d'une manière spécifique à modifier la forme générale de la trame urbaine en place, accentuant la fragilité de ces petits centres.

L'observation de ces deux cas particuliers tend donc à rappeler, qu'en dépit de conditions technologiques complètement différentes, la récession d'une branche ne frappe pas de manière aléatoire ce support géographique privilégié qu'est la trame urbaine. Quand la main-d'cuvre est le facteur de production le plus important les logiques qui guident cette altération paraissent extrêmement dépendantes de l'organisation hiérarchique de cette trame. Si, comme nous le proposions au début de cet exposé, ces activités en déclin ont un peu anticipé sur les processus aujourd'hui à l'action dans l'évolution du réseau des villes, toute action d'aménagement conduite à cette échelle devrait tenir compte de l'action de ces forces nouvelles. Interrompant le lent mouvement de translation, c'est-à-dire de transformation largement commune à toutes les villes qu'avait provoqué la croissance des décennies antérieures, ces forces tendraient aujourd'hui à secréter certaines disparités interurbaines, à en approfondir de plus anciennes ou certaines plus récentes, mais que l'on pouvait considérer jusqu'ici comme encore mineures.

Nos conclusions ont certes les limites de l'échantillon à partir duquel elles ont été formulées. Pourtant, les résultats auxquels nous sommes parvenues par la suite, en travaillant sur un échantillon beaucoup plus vaste de villes, ont eu tendance à les confirmer et à en élargir la portée. 


\section{DISCUSSION}

M. FÉNELON : La distance à Paris a-t-elle influencé l'évolution des industries navales ? La hiérarchie urbaine dans la France de l'Ouest n'a-t-elle pas été modifiée par l'industrialisation? n'y a-t-il pas des irrégularités, des disparités dans l'influence exercée.

M"e SAINT-JULIEN : La distance à Paris n'a jouré un rôle que dans l'évolution des industries en croissance rapide. Dans le cas de la construction navale, les structures anciennes ont été déterminantes.

Dans l'étude on s'est prioritairement intéressé aux régularités qui permettent de saisir et de comprendre les mécanismes essentiels.

M. GEORGE : La période étudiée a été extrêmement riche en modifications technologiques, en transformations de la structure et des coefficients d'emploi et ceci à l'intérieur même de chaque branche industrielle, il s'en est suivi une modification des comportements géographiques. Cependant il convient de considérer de façon distincte les différentes fonctions existant dans l'industrie. En ce qui concerne les fonctions de direction et de gestion, malgré les progrès de la télématique, la tendance fondamentale reste celle de la concentration. 